\title{
Harmonic and Resonance Analysis of High-speed Train-network Coupling Systems Based on Harmonic Admittance Matrix Model
}

\author{
Bohong Li, Fei Lin and Mengyue Zhou \\ College of Electrical Enginering of Beijing Jiaotong university, Beijing 100044, \\ China.xiaoxingxing_123@sina.cn
}

\begin{abstract}
To study the impact of fundamental flow and background harmonics on harmonic and resonance characteristics of high-speed train-network coupling systems, harmonic admittance matrix model for four quadrant converter is deduced with the method of FFT and Bessel function. Equivalent load of train and chain network model of multiple conductors was established to conduct the calculation of fundamental and harmonic flow. Based on all of these, realize the harmonic and resonance research of train-network coupling system with multiple trains, which offer the reference to safe operation and avoid the voltage peaks in actual supply network.
\end{abstract}

Keywords: harmonic admittance matrix, train-network coupling, power flow calculation, multiple conductor, harmonic and resonance

\section{Introduction}

The modern high-speed trains generally use four quadrant converters in the AC-DC-AC drive system. Although it obtains the higher power factor compared with traditional rectifier device and reduces the low frequency harmonics of AC side greatly, it may cause damage of electrical equipment, interference of communication systems because of the fast switching action. The problems become the major hidden danger affecting the safe operation of the railway.

At present, the study of high frequency harmonics of traction power supply system is mainly divided into two directions. From the perspective of the train, the study focus on the harmonic characteristics of four quadrant converter under different operating conditions, control strategy, multiple technologies and other different factors. From the perspective of the traction power supply system, establish multiple-conductor chain network model [1] for power supply network and trade the train's equivalent load as power source, voltage source or current source. Based on all of these, researchers study the effect of train on the harmonics and resonance in traction network. With the development of the research, in order to obtain a better reflection of electrical parameters' variation in the process of train running, the research is no longer limited to the independent research of train or traction network. Researchers put more emphasis on the effect of mutual coupling between trains and network. Such as paper [2] regard train as a constant power load model in the traction system, put forward an iteration algorithm for power flow calculation to study the voltage distribution. This paper provides a basis for the calculation of fundamental power flow in traction network. But there's no accurate coupling process involved in this paper. With the development of further research, the reference [3] put forward that if There is harmonic voltage in power grid, harmonic current of ac side of four-quadrant converter will be influenced, and then affect the coupling phenomenon between load and network. It provides the new thought of studying the coupling system of trains and traction nets. 
Therefore, for the high frequency harmonics and resonance analysis of the coupling system of trains and traction nets, the harmonics generated by the traction drive system of trains inject into traction nets and change its harmonic voltage distribution. This will finally become the background harmonics of other trains on the same supply range. To study the influence of background harmonics on four quadrant converter, this paper put forward the harmonic admittance matrix which can map all the odd harmonic voltages to the harmonic currents by one matrix. In the past study of trains, the high-speed train is generally traded as harmonic source which was modeled as voltage source, constant current source, power source and so on. Though these models can reflect the harmonic characteristics in the process of train running to some extent, they don't involve the influence of power flow and background harmonics.

As the simplest general harmonic source model, constant current source only depends on the fundamental voltage imposed on trains, and the equivalent impedance of train is approximately infinite for background harmonics. However, though the harmonic voltage is much smaller than fundamental voltage, it will reduce the accuracy of the simulation if harmonic voltage is ignored. In addition, reference to the model of nonlinear load in electric system, there are Norton model, harmonic coupling admittance matrix model and harmonic source model based on least squares approximation. Though the Norton model reflects the combination of harmonics and fundamental, but now it is generally obtained by measuring method. For the harmonic coupling admittance matrix model [4], its accuracy depends on the admittance matrix and the calculation process is extremely complex as well as harmonic source model based on least squares approximation. The complexity makes them difficult to get practically applied.

Paper [5] uses the methods of measuring to get harmonic admittance matrix model of arbitrary nonlinear load. Paper [6 8] with methods of differential equations of circuit, FFT analysis and the integration, respectively get the harmonic admittance matrix models of single phase uncontrolled and controlled rectifier circuit, which provides reference for this paper's theoretical derivation. The four quadrant converter is generally used in the high-speed train, and its main difficulty is that the switching function $s(t)$ is multi-state and nonlinear and its Fourier analysis is very complicated. At present, there is no relevant article has clearly deduced the coupling harmonic admittance matrix model adapted to four quadrant converter.

On the basis of train and supply line model, to study the influence of traction nets power flow, this paper put forward the calculation method of fundamental and harmonic power flow which formed the architecture of simulating the coupling system. Firstly, this article conducted the theoretical analysis of four quadrant model and deduced the harmonic admittance matrix under the condition that there is background harmonics at the AC side. Then, account for the multiple-conductor chain network model of the traction power supply system and iterative algorithm of power flow. In the simulation part, first part is the verification of the harmonic admittance matrix. Then under considering the influence of fundamental and background harmonic, the last part analysis the harmonics and resonance characteristics of coupling system by using harmonic admittance matrix the model.

\section{Harmonic Admittance Matrix}

This paper referring to the derivation method of crossover frequency coupling admittance matrix mode for uncontrolled rectifiers in electrical system, deduced the four quadrant converter harmonic admittance matrix model to reflect the influence of background harmonic voltage on the four quadrant harmonics. Besides, the model is validated by comparing the theoretical results with Simulink model results of four quadrant converter. 


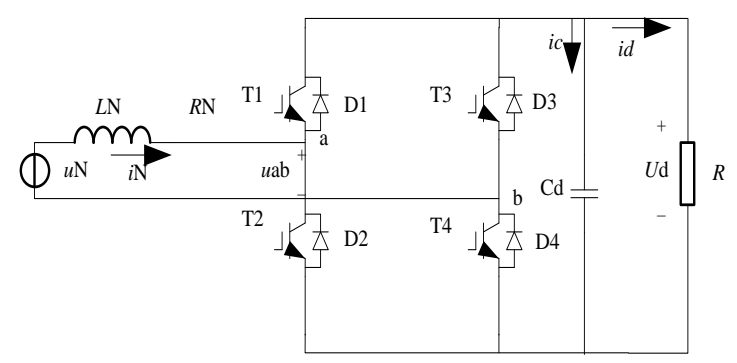

Figure 1. The Single Phase Two Level Four Quadrant Converter

As shown in Figure 1, it is the schematic diagram of single phase two level four quadrant converter. $u_{N}$ is the AC side voltage, $L_{N}$ and $R_{N}$ is equivalent inductance and resistance, $C_{d}$ is the DC side support capacitor, $R$ is the load, $U_{d}$ is the DC side voltage. PWM modulation signal generated by the control part of the rectifier will compare with carrier signal to get pulse control signal of both bridge a and bridge $b$. The pulse control signal regulate the four switches to realize the rectifier and inverter [9 12]. The simplified equivalent switching circuit is as follow in Figure 2.

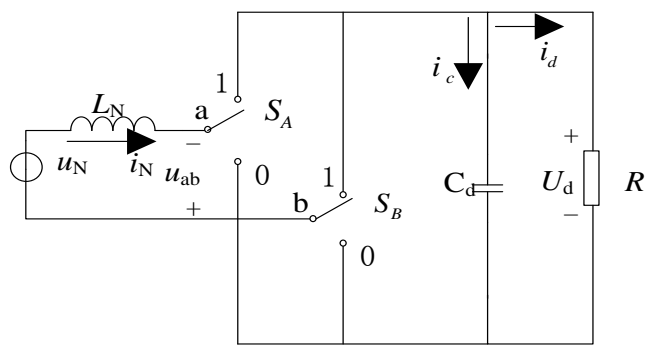

\section{Figure 2. The Equivalent Switching Circuit of Level Four Quadrant Converter}

Switching function SA and SB of bridge a and bridge $b$ changes to 1 or 0 with the state changing of switches. Specific correspondence is as below:

$$
\begin{gathered}
S_{A}\left\{\begin{array}{lcc}
1 & T_{1} \text { and } D_{1} & \text { closed } \\
0 & T_{2} \text { and } D_{2} & \text { open }
\end{array}\right. \\
S_{B}\left\{\begin{array}{lcl}
1 & T_{3} \text { and } D_{3} & \text { closed } \\
0 & T_{4} \text { and } D_{4} & \text { open }
\end{array}\right.
\end{gathered}
$$

$S(t)$ is the ideal switch function between point $\mathrm{a}$ and $\mathrm{b}$ at the combined effect of switch SA and SB. So, it can be written as:

$$
u_{a b}(t)=(S A-S B) \times u_{d c}(t)=s(t) \times u_{d c}(t)
$$

In order to simplify the derivation process, assuming that $u_{d c}(t)$ is a fixed value, which means $U_{d c}=u_{d c}(t)$. The assumption has been verified in reference [5], which will not affect the harmonic analysis. According to the equation (1), the four quadrant converter circuit is further simplified as shown in Figure 3: 


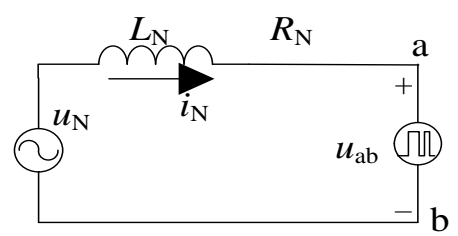

Figure 3. Simplified Circuit of Four Quadrant Converter

Thus, the differential equation is gained as follow:

$$
u_{N}=L_{N} \frac{d i_{N}}{d t}+u_{a b}
$$

Ideally, $u_{N}$ is the fundamental voltage, but in the actual systems, $u_{N}$ contains a number of odd harmonics, so here we define the voltage source as below:

$$
u_{N}(t)=\sum_{h=1}^{H} U_{h} \cos \left(h \omega t+\phi_{h}\right)
$$

Meanwhile, due to there are a number of odd harmonics existed in $i_{N}$, so the assumption for the current of $\mathrm{AC}$ side is:

$$
i_{N}(t)=\sum_{h=1}^{H} I_{h} \cos \left(h \omega t+\gamma_{h}\right)
$$

$U_{a b}$ is the PWM pulse voltage obtained from the difference between $u_{a}(\mathrm{t})$ and $u_{b}(\mathrm{t})$ after modulation, and its modulation process is shown in figure 4 as follow:
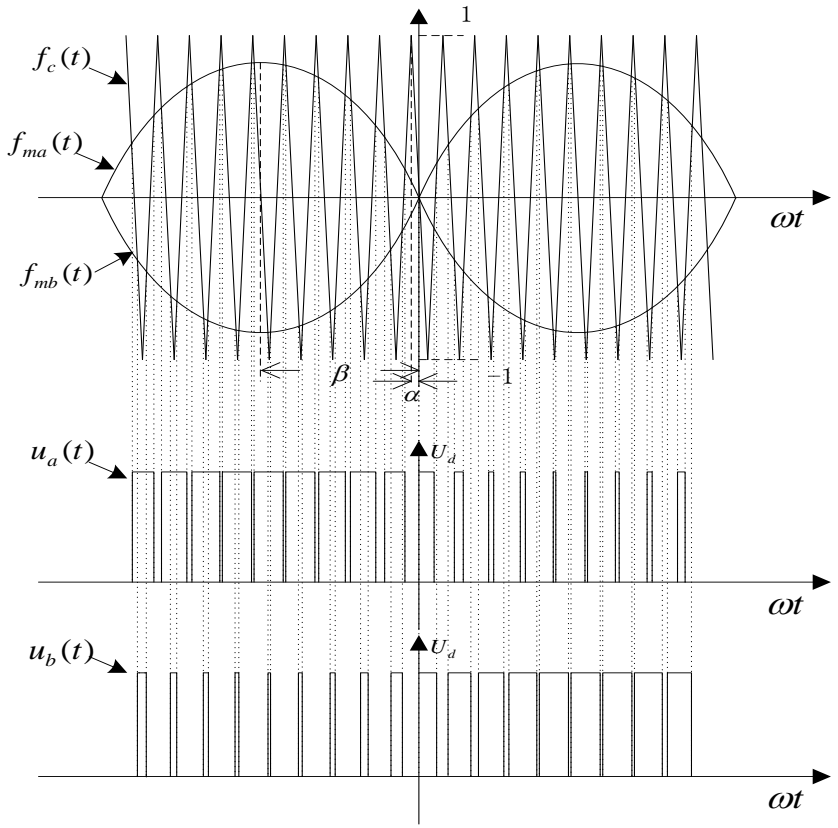

Figure 4. Principle of Four Quadrant Converter Modulation

$f_{m a}$ and $f_{m b}$ is the modulating signal of phase a and $\mathrm{b}$, whose expression is :

$$
\begin{aligned}
& f_{m a}(t)=M \cos \left(\omega_{m} t+\beta\right) \\
& f_{m b}(t)=-M \cos \left(\omega_{m} t+\beta\right)
\end{aligned}
$$

$M$ is the modulation depth, 
$\omega_{m}$ is the modulation frequency,

$\beta$ is the modulation initial phase angle.

$f_{c}(t)$ is the carrier signal of phase a and $\mathrm{b}$, its expression is:

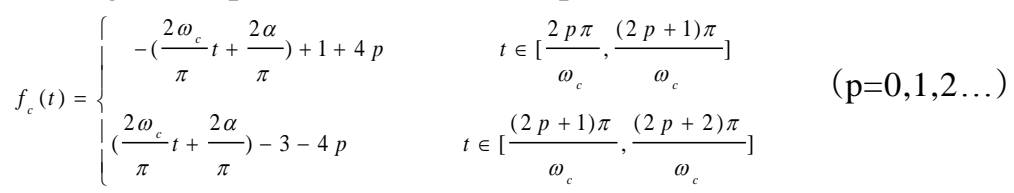

$\mathrm{c}$ is carrier frequency,

$\alpha$ is the carrier initial phase angle.

When $f_{m a}(\mathrm{t})>f_{c}(\mathrm{t}), u_{a}(\mathrm{t})=U_{d}$, make $x=\omega_{c} t, y=\omega_{m} t$.

Rising edge appears at:

$$
1+4 p-\left(\frac{2 \omega_{c}}{\pi} t+\frac{2 \alpha}{\pi}\right)=M \cos \left(\omega_{m} t+\beta\right)
$$

After calculation:

$$
x=\frac{\pi}{2}[1-M \cos (y+\beta)]+2 p \pi-\alpha
$$

Falling edge appears at:

$$
-3-4 p+\left(\frac{2 \omega_{c}}{\pi} t+\frac{2 \alpha}{\pi}\right)=M \cos \left(\omega_{m} t+\beta\right)
$$

After calculation:

$$
x=-\frac{\pi}{2}[1-M \cos (y+\beta)]+(2 p+2) \pi-\alpha
$$

Use the FFT analysis at PWM pulse signal within a modulation cycle, then the voltage expression of bridge a is deduced as $u_{a}(t)$. The modulation wave of phase $\mathrm{b}$ is opposite to a, which means the difference of phase is $180^{\circ}$. Therefore, rewritten the modulation initial phase $\beta$ as $\beta+\pi$, then $u_{b}(t)$ which is the voltage expression of bridge $\mathrm{b}$ is obtained. The two expressions are as below:

$$
\begin{aligned}
u_{a}(t)= & \frac{U_{d}}{2}+\frac{M U_{d}}{2} \cos \left(\omega_{m} t+\beta\right)- \\
& \sum_{m=1}^{\infty} \frac{2 U_{d}}{m \pi} \sin \frac{m \pi}{2} J_{0}\left(\frac{m \pi M}{2}\right) \cos \left(m \omega_{c} t+m \alpha\right)- \\
& \sum_{m=1}^{\infty} \sum_{\substack{n=-\infty \\
n^{10}}}^{\infty} \frac{2 U_{d}}{m \pi} \sin \left[(m-n) \frac{\pi}{2}\right] J_{n}\left(\frac{m \pi M}{2}\right) \cos \left(m \omega_{c} t+n \omega_{m} t+m \alpha+n \beta\right) \\
u_{b}(t)= & \frac{U_{d}}{2}-\frac{M U_{d}}{2} \cos \left(\omega_{m} t+\beta\right)- \\
& \sum_{m=1}^{\infty} \frac{2 U_{d}}{m \pi} \sin \frac{m \pi}{2} J_{0}\left(\frac{m \pi M}{2}\right) \cos \left(m \omega_{c} t+m \alpha\right)- \\
& \sum_{m=1}^{\infty} \sum_{\substack{n=-\infty \\
n \neq 0}}^{\infty} \frac{2 U_{d}}{m \pi} \sin \left[(m+n) \frac{\pi}{2}\right] J_{n}\left(\frac{m \pi M}{2}\right) \cos \left(m \omega_{c} t+n \omega_{m} t+m \alpha+n \beta\right)
\end{aligned}
$$

$J_{n}$ is the Bessel function, $\mathrm{n}$ is the order.

Then get the AC side voltage expression $u_{a b}(t)$ : 
International Journal of $u$ - and e- Service, Science and Technology

Vol.8, No.1 (2015)

$$
\begin{aligned}
u_{a b}(t)= & u_{a}(t)-u_{b}(t) \\
= & M U_{d} \cos \left(\omega_{m} t+\beta\right)+ \\
& \left.\sum_{m=2,4 \ldots n= \pm 1, \pm 3 \ldots}^{\infty} \sum_{n}^{\infty} \frac{4 U_{d}}{m \pi} \sin \frac{n \pi}{2} \cos \frac{m \pi}{2} \omega_{m} t+m \alpha+n \beta\right)
\end{aligned}
$$

To obtain M, firstly assume the active power of four quadrant converter is $\mathrm{P}$, and then draw the fundamental vector diagram of converter in Figure 5:

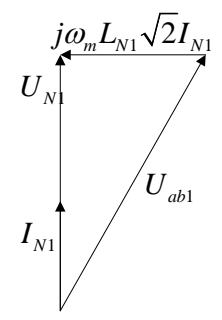

\section{Figure 5. Fundamental Vector Diagram under Traction Condition}

Thus, on the basis of balance of the fundamental active power and reactive power, get the equations as:

$M$ is obtained as:

$$
\left\{\begin{array}{c}
P=\frac{U_{N} M U_{d}}{\omega_{m} L_{N} \sqrt{2}} \sin \beta=I_{N} U_{N} \\
M U_{d} \cos \beta=\sqrt{2} U_{N}
\end{array}\right.
$$

$$
M=\frac{\sqrt{2} \sqrt{U_{N}{ }^{4}+\left(P \omega_{m} L_{N}\right)^{2}}}{U_{N} U_{d}}
$$

Take the formula (3), (4) and (14) into (2), finally the harmonic admittance matrix can be deduced as formula (17) after a series of consolidation.

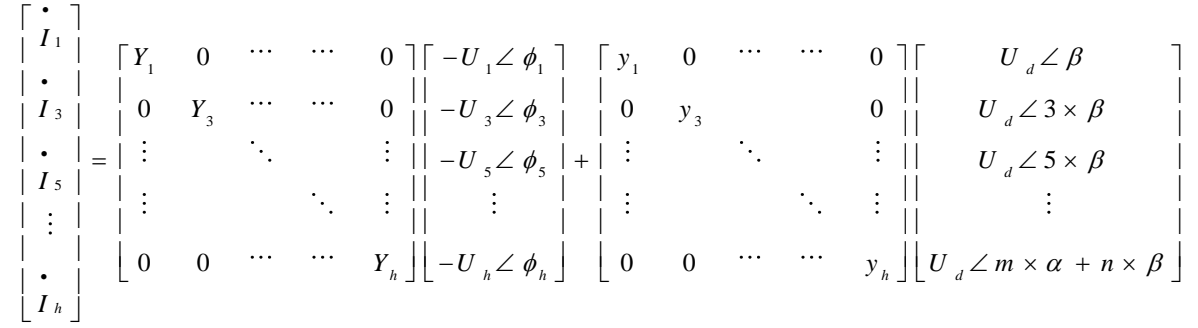

In the formula:

$$
\left\{\begin{array}{c}
Y_{h}=\frac{1}{h \omega_{m} L} \\
y_{h}=\frac{4}{h m \pi \omega_{m} L} \sin \left(\frac{n \pi}{2}\right) \cos \left(\frac{m \pi}{2}\right) J_{n}\left(\frac{m \pi M}{2}\right)
\end{array}\right.
$$$$
h=3,5 \cdots
$$ 


\section{System Modeling and Power Flow Calculation}

\subsection{Power Flow Calculation}

We can observe the harmonic amplification and resonant phenomenon by power flow calculation which is able to detect the voltage of each node in the traction nets. The harmonic power flow stems from the fundamental power flow and the high frequency harmonic source is produced by nonlinear characteristics of four quadrant converter. The two reasons make the harmonic transfer from the position of train to both ends of the railway. This transmitting procedure is the harmonic power flow. Due to the fundamental having great influence on the harmonic network parameters, but harmonics have little influence on fundamental, so the calculation results of fundamental power flow is always regarded as the input of harmonic power flow when considering the coupling effect between fundamental and harmonics. Usually, the fundamental current is a function of fundamental voltage and harmonic voltage. Further, complete the power flow calculation through iterative method. The figure 6 is the calculation block diagram of fundamental and harmonics power flow, where $U_{n k}$ is the node voltage and $U_{h k}$ is the harmonic voltage.

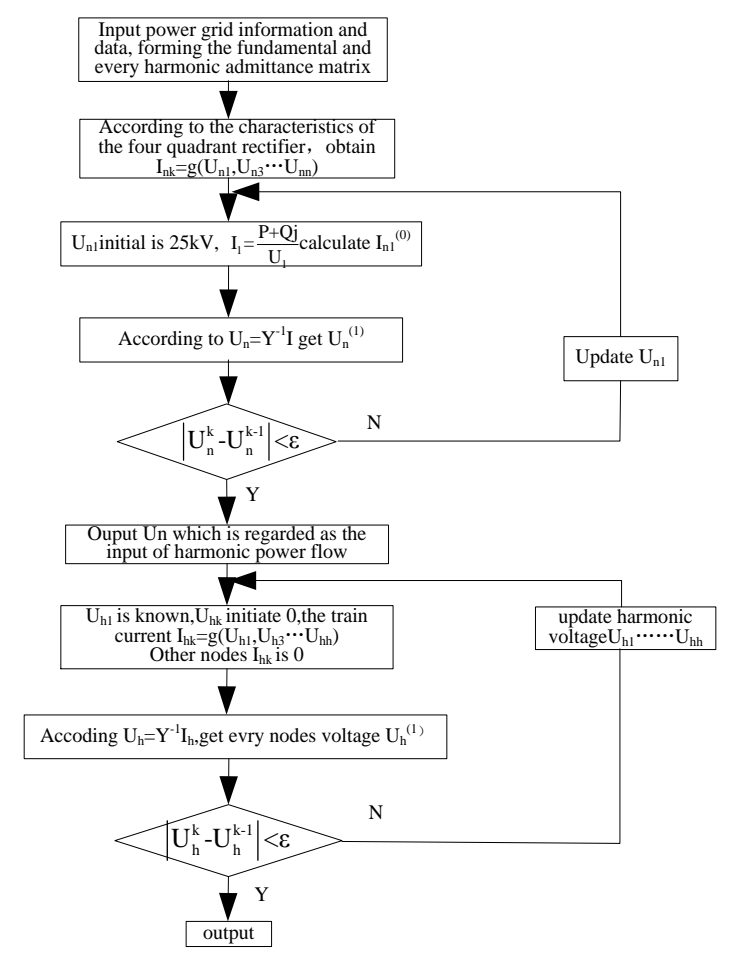

Figure 6. Flow Diagram of Power Flow

Paper [1] regards the high-speed train as the constant power load when studying power flow of the train-network coupling systems, and it holds that when a train is in constant power consumption, its equivalent impedance changes along with the network voltage. This kind of equivalent method in this paper also takes this thinking. It is different from constant impedance and constant current model because the constant power source involves the mutual influence between multiple trains running in the lines. However, because of the mutual influence, the fundamental and harmonic voltage and current vectors of each node on the power supply nets must be realized by iteration algorithm. What calls for special attention is 
that in harmonic power flow, the harmonic current is decided by both fundamental voltage and harmonic voltage and the function is nonlinear. So from the above, the harmonic admittance matrix model with iteration algorithm is adopted in this paper to obtain the harmonic voltage distribution of the power supply nets.

\subsection{Power Supply System}

At present, the high-speed train traction network has widely used AT power supply model with autotransformer, whose characteristics is that every 10 to 15 kilometers there is an autotransformer connected in parallel between catenary and negative feeder with middle tap connected to the rail [13]. The traction network is a parallel multi-conductor transmission system made up of supply and backflow network wires, and it can be modeled as chain network topology. To simplify the calculation without affecting the accuracy of model, 12 conductors usually be replaced by the equivalent model of 5 conductors [14-17]. Every unit length of 5 conductors circuit is seen as a $\pi$ type equivalent circuit, in which $Z_{L}$ and $Y_{L}$ are all 5 -order square matrix. On this basis, multiple $\pi$ type equivalent circuits cascade together and form the chain network model of the whole traction nets. Every unit length of the chain network model can be a section. On the chain network model system, the autotransformer, parallel resistance and other cross-links are equivalent to 5-order admittance matrix, and a train is equivalent to a five-dimension vector. The components aforesaid is in parallel in the chain network to perfecting the system modeling. As shown in Figure 7, all the planes are seen as nodes. Then, establish node voltage equation to obtain voltage distribution of traction nets.

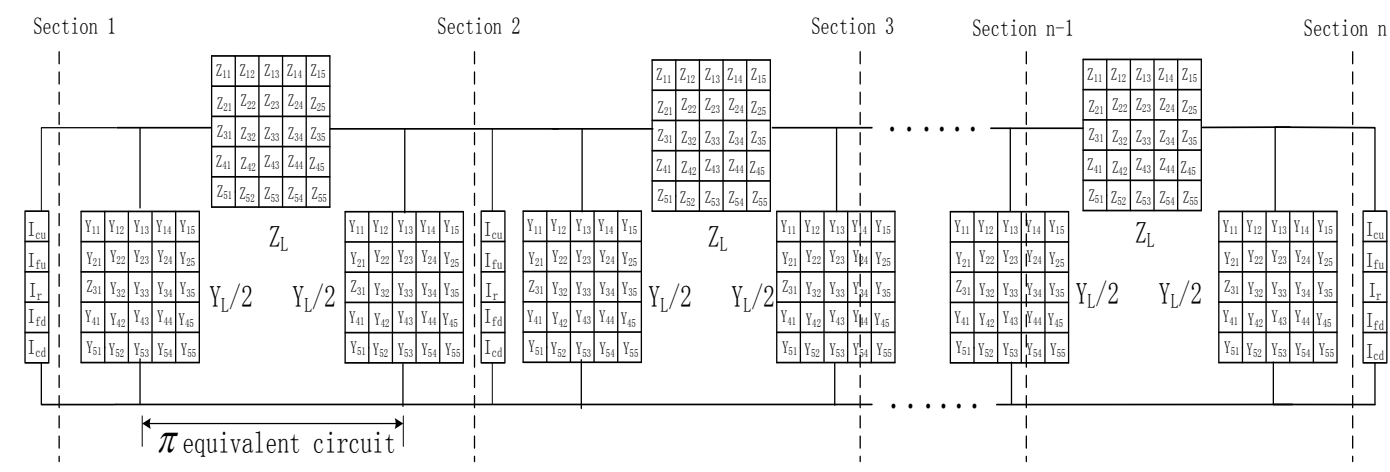

Figure 7. 5-conductor Chain Network Model

After repeated simulation tests, it is found that the accuracy of AT transformer has great influence on harmonic flows. In order to reflect the harmonic voltage distribution more objective, this paper assumes that the AT transformer is an ideal autotransformer in the flow calculation and then gets the voltage distribution through current distribution of traction network. As shown in Figure 8, the left and right side of imaginary line is respectively the upgoing and down-going lines of traction nets. Due to the current flows mainly through the catenary and negative feeder, so the double track AT supply nets can be equivalent to two side feeding nets of single line. 


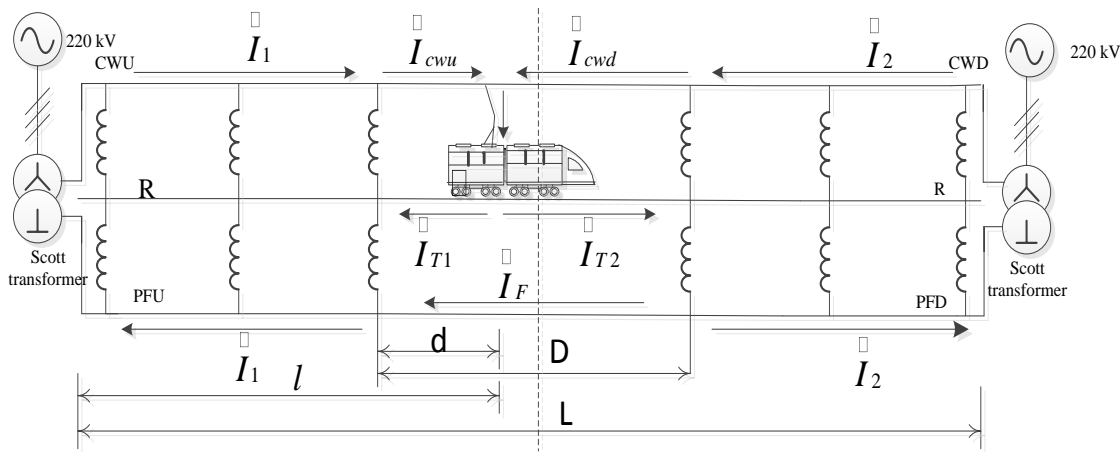

Figure 8. Current Distribution of Double-tracking AT Network

\section{Simulation Result}

\subsection{Verification of Harmonic Admittance Matrix}

In order to verify the correctness of model, this paper add harmonic sources in different orders to the AC side of Simulink model of for four quart converter, and then conduct FFT analysis on the AC side. To comparing the results between simulation and theory, the correctness of harmonic admittance matrix is verified. Figure 9 shows the FFT analysis result of $\mathrm{AC}$ side current at the $1250 \mathrm{~Hz}$ switching frequency without additional harmonic voltage sources at the AC side.

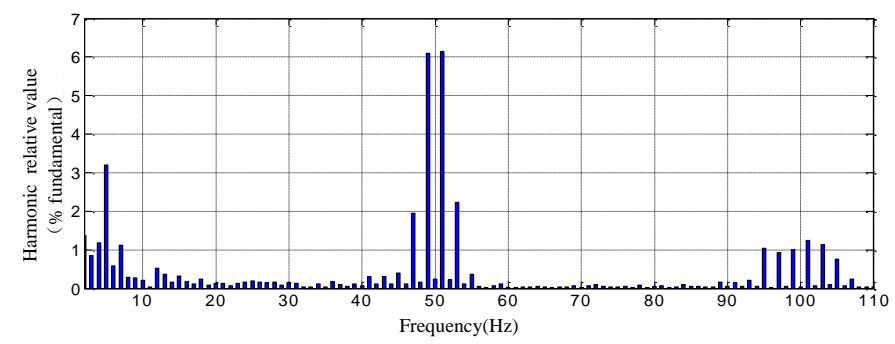

\section{Figure 9. FFT Analysis Results of Four Quadrant Converter AC Current}

It can be seen in the Figure 9 that characteristic harmonics are mainly concentrated in 2 and 4 times near the switching frequency. At the AC side of Simulink model, add certain harmonic voltage source with the amplitude which is the 10 percent of fundamental amplitude and measure the harmonic current at the $\mathrm{AC}$ side. On the other hand, take the corresponding harmonic voltage to formula (17) and measure the harmonic current as well. Therefore, the contrast results of simulation and theory is shown in Figure10.

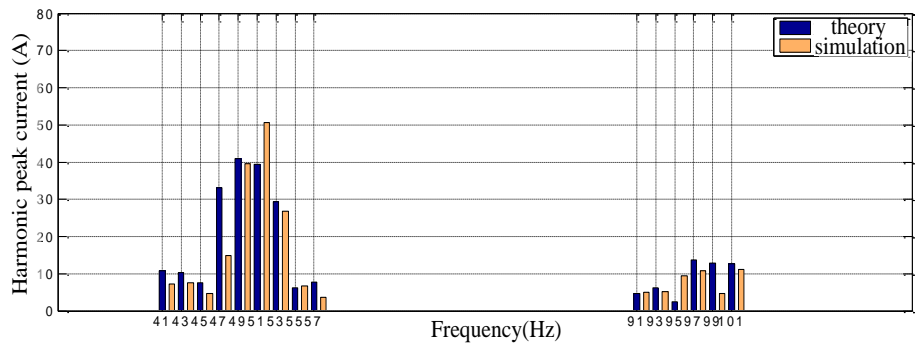

Figure 10. Harmonic Comparison of AC Current 
The Figure 10 shows that the simulation result and theoretical derivation are basically the same, which means the harmonic admittance matrix is feasible when used in studying the influence of background harmonics on four-quadrant converter.

\subsection{Analysis of Example}

In order to study the influences of background harmonic and other factors on the harmonics and resonance of traction nets, take a power supply arm of one substation as as example. This is shown in Figure 11. The rated power of train is $2 \mathrm{MW}$. On the basis of this example, the lateral comparison was made among the factors such as fundamental flow, harmonic flow and multiple trains on running. Furthermore, the vertical comparison was also made to research the phenomenon of harmonics, resonance and the distribution of harmonic voltages.

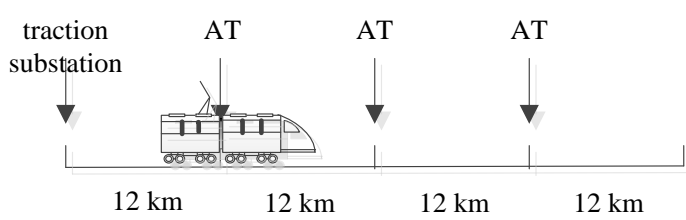

Figure 11. Example of Power Supply Network

4.2.1. Impact of Fundamental Flow and Background Harmonics on the Running Train: Assuming a train running at the position of $12 \mathrm{~km}$ far from substation, the ideal voltage distribution curve of $99^{\text {th }}$ harmonic was drawn. When considering the fundamental flow which makes the voltage is not the rated $25 \mathrm{KV}$, draw another voltage distribution curve. Furthermore, to involve the influence of harmonic flow, assume there is harmonic source at the position of $48 \mathrm{~km}$ which will change the harmonic voltage at $12 \mathrm{~km}$. So based on the harmonic admittance matrix model, gain the third curve under the background harmonics as shown in Figure 12.

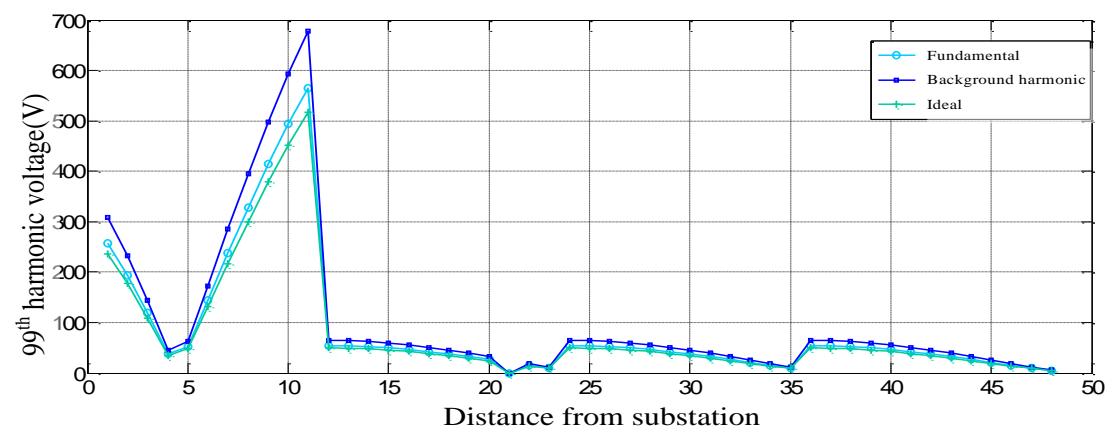

\section{Figure 12. Harmonic Comparison for AC Current under Different Conditions}

Through the picture, we can see the fundamental and background harmonics do not change the position of the resonance peaks but have the amplitude reduced. Theoretically, if the train is a power source, with the increase of voltage the current will decrease and its impact in the power supply nets is weaker. The result shown in the picture is consistent with theory. 
Extending the simulation to $41 \sim 99^{\text {th }}$ harmonic, the harmonic voltage distribution with and without background harmonics in supply network were shown in Figure 13 and Figure 14.

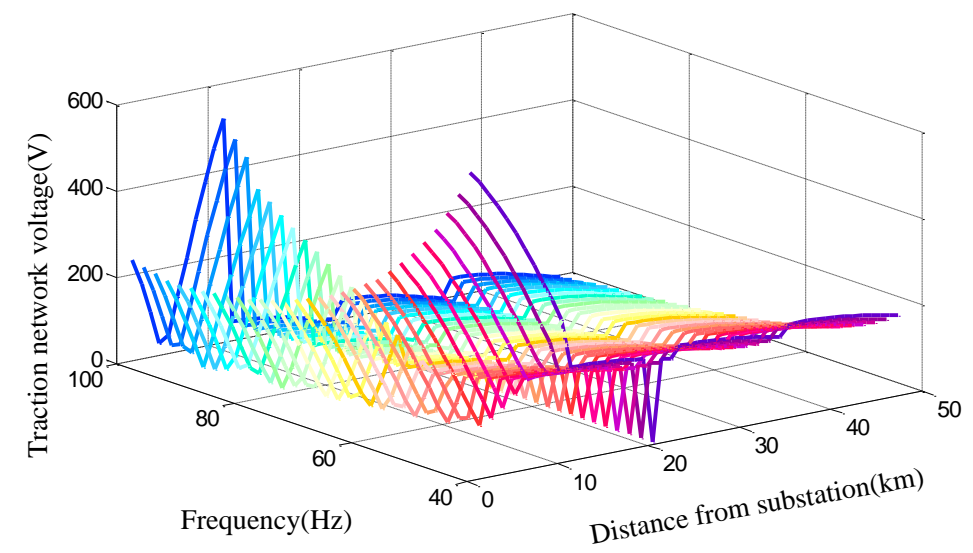

Figure 13. Voltage Distribution under Ideal Condition

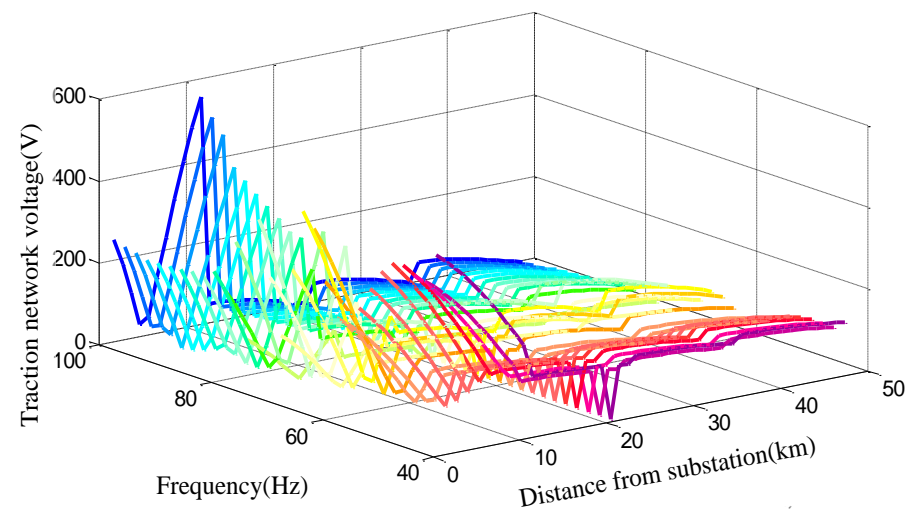

Figure 14. Voltage Distribution under Background Harmonics

The voltage peaks of different harmonics are not the same, and the influences of background harmonics on the different harmonics are different as well. The voltage peak of harmonics whose frequency is near the four times of switching frequency appears near the position of $10 \mathrm{~km}$ far from the substation, and its voltage distribution is not obviously affected by background harmonics. However, the voltage peak of harmonics whose frequency is near the two times of switching frequency appears near the substation, and its voltage distribution showed a trend of decrease under the effect of background harmonics. The same influence between fundamental flow and background harmonics is that they all have nothing to do with the positon of peaks. The high voltages is mainly occurs between two AT substations near the harmonic source. With the spread of the harmonic current, the harmonic voltages change periodically in other supply sections far from train. It can be inferred that the admittance and impedance matrix are only related to the harmonic frequency, which means the simulation result agrees with the theory. 
4.2.2. Impact of Fundamental Flow and Background Harmonics on Multiple Trains: In the real system, there may be multiple trains running at the same time. All the trains are equivalent to harmonic sources which will inject into the traction network and become background harmonics of each other. So, the harmonic voltage distribution should be the superposition of instantaneous data from different trains. Considering from the aspect of algorithm realization, it is better to apply iterative algorithm when studying the influence of background harmonics on multiple trains.

In Figure 15, we get the $51^{\text {th }}$ harmonic voltage distribution of two trains respectively without considering mutual influence. Assume the two trains were all running on the upgoing lines. One is at the position of $12 \mathrm{~km}$, and the other is at the position of $48 \mathrm{~km}$.

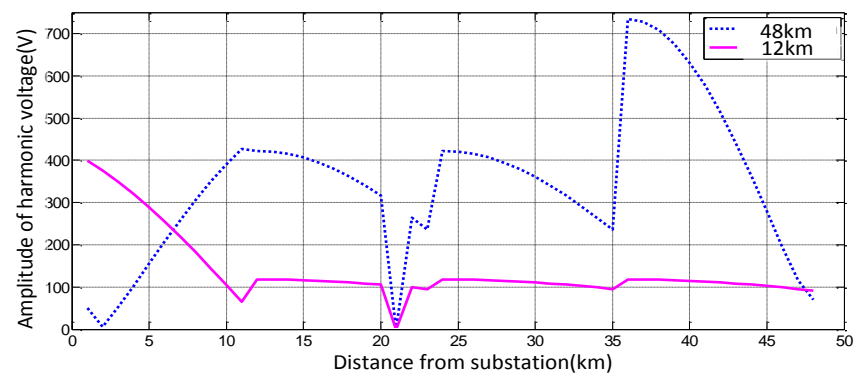

Figure 15. Harmonic Distribution of Different Trains

When considering the influence of multiple trains, the terminal condition of iterative calculation has guaranteed the convergent precision. Then, the new harmonic distribution was obtained as Figure16.

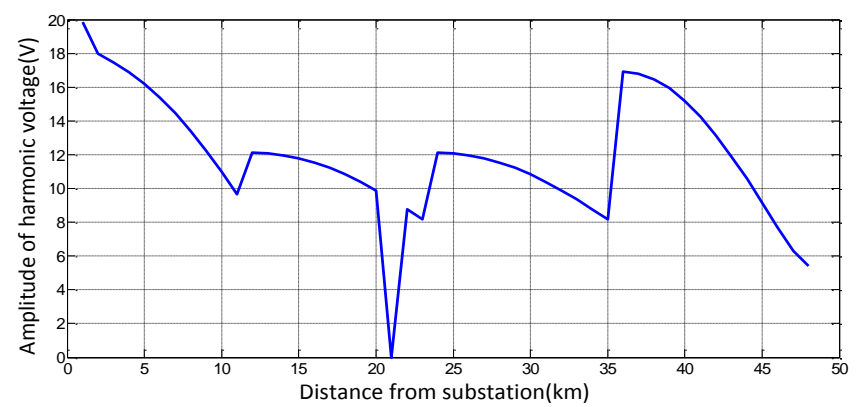

\section{Figure 16. Harmonic distribution with affection of Each Other}

It can be seen that the whole harmonic voltage has decreased. It means the effect of background voltage makes the harmonic voltage generated by the equivalent currents of trains weaken. Meanwhile, under the interaction of each other, the harmonic voltage value of peaks has increased compared to isolated operation condition, and it seems like the superposition of the previous peaks.

When there is one train on both up-gong lines and down-gong lines, assume the two trains both running at the position of $12 \mathrm{~km}$ from substation. Figure 17 shows the respective voltage distribution without considering mutual influence. 


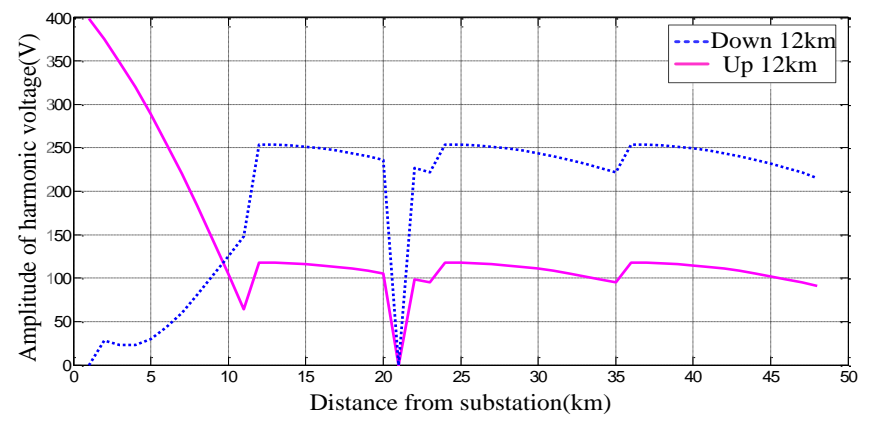

Figure 17. Harmonic Voltage Distribution of Different Trains

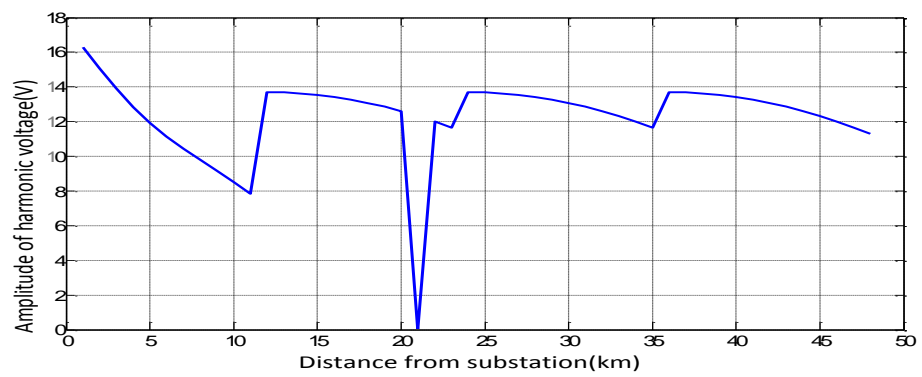

Figure 18. Harmonic Distribution with Affection of Each Other

The Figure 18 shows the $51^{\text {th }}$ harmonic voltage distribution with the mutual influence of two trains. The positions of the resonant peaks still remain unchanged and the value is reduced like previous. What is different from the simulation result of the previous up-going trains is that they compromise at the peaks instead of superposition.

\section{Conclusion}

Based on the power flow calculation and harmonic admittance matrix model, the trainnetwork coupling simulation provides assistance to the study of high frequency harmonics and resonance of traction power supply systems. The conclusions are as below:

(1) The effect of fundamental flow and background harmonics does not change positions of the resonant voltage peaks. The admittance and impedance matrix are only related to the harmonic frequency.

(2) The effect of fundamental flow and background harmonics reduces the peak value of harmonic voltages. When the train is treated as power source, with the increase of voltage, the current will decrease and its impact on the power supply network is weaker.

(3) Harmonic voltage peaks of different high harmonics occur in different places. The same value of different harmonic background voltages has different degree of influence. From the respect of simulation results, compared with four times switching frequency, the harmonics near the two times switching frequency are affected more seriously.

(4) Harmonic voltage peaks mainly occur between the two AT autotransformer station nearest to the train, and the harmonic voltages change periodically in other supply sections far from train.

(5) When there are multiple trains running, the value of harmonic voltage distribution will decrease under the influence of multiple background harmonics. If the trains run in the 
same direction, under the interaction of each other, the harmonic voltage value of peaks will be the superposition of the previous peaks. However, if the trains run in different directions, the value will compromise at the peaks instead of superposition.

According to the conclusions of the simulation, some operation instructions can be given. Conclusion (1)(2)(3) can help obtain the resonant frequency and the voltage peaks, so in the real system, a detector can be installed in the train to detect the frequency and voltages, then analyze the information with the method of simulation aforementioned to predict the potential threats of resonance. Based on the prediction, change the four quadrant control system to avoid the harmonic frequency. In addition, when the trains running at the same direction, the conclusion (4)(5) indicates that it is better to avoid the phenomenon that multiple trains running at the same two adjacent AT autotransformer stations. However, if the distribution of trains not only in up-track but also in down-track, it is unnecessary to avoid this kind of phenomenon.

This paper is just the preliminary study of the train-network coupling system. Because of the complexity of the coupling system, many influence factors such as the relative positions of trains, different drive system and different switching frequency need to be quantitatively analyzed in the future study. In addition, the prediction technique mentioned in the previous paragraph is still need to be realized in further step.

\section{Acknowledgements}

This work was supported in part by teachers of Beijing Jiaotong University and the classmates in Yang \& Lin Lab. Most of the reading materials are obtained by IEEE, which offers the important information and reference for this study. In addition, it is very grateful for the research of $\mathrm{Xi} \mathrm{Chu}$ and Mingli Wu who provide the solid foundation for further study of train-net coupled systems.

\section{References}

[1] R. J. Hill, S. Brillante and P. J. Leonard, "IEEE Proc. Electr. Power Appl.”, vol. 1, no. 146, (1999).

[2] H. T. Hu, Z. Y. He and J. F. Wang, "Proceedings of the Chinese Society for Electrical Engineering", vol. 19, no. 32, (2012).

[3] W. Fang, X. Li and L. Xu, "Chinese Journal of Power Sources", vol. 7, no. 37, (2013).

[4] X. H. Wang and X. M. Yu, "Electrotechnical Application", vol. 2, no. 27, (2008).

[5] M. Fauri, "Power Systems IEEE Transactions on", vol. 4, no. 12, (1997).

[6] J. Yong, L. Chen and S. Y. Chen, "Proceedings of the CSEE", vol. 25, no. 30, (2010).

[7] J. Yong, L. Chen, A. B. Nassif and W. Xu, "Power Delivery IEEE Transactions on", vol. 2, no. 25, (2010).

[8] Y. Y. Sun, G. B. Zhang and W. Xu, "Power Systems IEEE Transactions on", vol. 4, no. 22, (2007).

[9] X. L. Ge, X. Y. Feng and B. Liu, "Power Electronics", vol. 4, no. 43, (2009).

[10] M. Zhang, Z. Y. He, H. T. Hu, S. Wu and S. B. Gao, "Power System Technology", vol. 9, no. 36, (2012).

[11] B. J. Wu and F. Zhao, "Electrical Drive Automation", vol. 2, no. 34, (2012).

[12] X. Chu, F. Lin, Z. P. Yang and H. Sun,." Transactions of China Electrotechnical Society”, vol. S2, no. 28, (2013).

[13] M. L. Wu, "Proceedings of the CSEE", vol. 28, no. 30, (2010).

[14] Z. Y. He, H. T. Hu, L. Fang, M. Zhang and S. B. Gao, "Proceedings of the CSEE", vol. 16, no. 31, (2011).

[15] H. T. Hu, Z. Y. He and M. Zhang, "Proceedings of the CSEE", vol. 13, no. 32, (2012).

[16] J. Q. Liu, Q. L. Zheng and Q. L. Yang, "Transactions of China Electrotechnical Society, vol. 4, no. 48, (2013).

[17] G. W. Chang, H. W. Lin, S. K. Chen, "Power Delivery IEEE Transactions on", vol. 2, no. 19, (2004). 


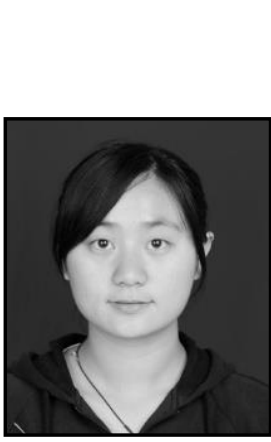

\section{Author}

Bohong Li, she got the bachelor's degree in Xi'an University of Technology in China. Recently she is studying in Beijing Jiaotong University for master degree. Her major is Electrical Engineering and Automation, and now she is primarily responsible for the research of harmonics in power supply system in Yang\&Lin Lab. 
International Journal of $u$ - and e- Service, Science and Technology Vol.8, No.1 (2015) 